\title{
Local Hydration Environments of Amino Acids and Dipeptides Studied by X-ray Spectroscopy of Liquid Microjets
}

\author{
B. M. Messer, ${ }^{\dagger, \sharp}$ C. D. Cappa, ${ }^{\dagger, *}$ J. D. Smith, ${ }^{\dagger, *}$ W. S. Drisdell, ${ }^{\dagger}$ C. P. Schwartz, \\ R. C. Cohen, ${ }^{\dagger}$ and R. J. Saykally $*, \dagger$, \\ Department of Chemistry, University of California, Berkeley, California 94720, and Chemical Sciences \\ Division, Lawrence Berkeley National Laboratory, Berkeley, California 94720
}

Received: July 11, 2005; In Final Form: September 13, 2005

\begin{abstract}
The nitrogen K-edge spectra of aqueous proline and diglycine solutions have been measured by total electron yield near-edge X-ray absorption fine structure (NEXAFS) spectroscopy at neutral and high $\mathrm{pH}$. All observed spectral features have been assigned by comparison to the recently reported spectrum of aqueous glycine and calculated spectra of isolated amino acids and hydrated amino acid clusters. The sharp preedge resonances at 401.3 and $402.6 \mathrm{eV}$ observed in the spectrum of anionic glycine indicate that the nitrogen terminus is in an "acceptor-only" configuration, wherein neither amine proton is involved in hydrogen bonding to the solvent, at high $\mathrm{pH}$. The analogous $1 \mathrm{~s} \rightarrow \sigma^{*}{ }_{\mathrm{NH}}$ preedge transitions are absent in the NEXAFS spectrum of anionic proline, implying that the acceptor-only conformation observed in anionic glycine arises from steric shielding induced by free rotation of the amine terminus about the glycine $\mathrm{CN}$ bond. Anionic diglycine solutions exhibit a broadened $1 \mathrm{~s} \rightarrow \pi^{*} \mathrm{CN}$ resonance at $401.2 \mathrm{eV}$ and a broad shoulder resonance at $403 \mathrm{eV}$, also suggesting the presence of an acceptor-only species. Although this assignment is not as unambiguous as for glycine, it implies that the nitrogen terminus of most proteins is capable of existing in an acceptor-only conformation at high $\mathrm{pH}$. The NEXAFS spectrum of zwitterionic lysine solution was also measured, exhibiting features similar to those of both anionic and zwitterionic glycine, and leading us to conclude that the $\alpha$ amine group is present in an acceptor-only configuration, while the end of the butylammonium side chain is fully solvated.
\end{abstract}

\section{Introduction}

Biochemical systems almost universally occur in aqueous environments. ${ }^{1}$ Accordingly, there has been a great effort made to understand the interplay between solvent and solute in biological molecules, particularly for proteins and the amino acids comprising them. NMR studies measuring the molecular weight dependence of $\mathrm{N}-14$ and $\mathrm{O}-17$ relaxation times have been used to determine the number of water molecules participating in hydrogen bonding with either the amine or carboxylic acid portions of amino acids, respectively. ${ }^{2,3}$ The results of these studies suggest that the cationic form is solvated by an excess of $1-3$ water molecules compared to the zwitterion. ${ }^{3}$ While no difference in O-17 relaxation times were observed between the zwitterion and anion, ${ }^{2}$ the $\mathrm{N}-14$ relaxation times were not measured at high $\mathrm{pH}$, thereby precluding any comparison between the $-\mathrm{NH}_{3}{ }^{+}$terminus of the zwitterion and the $-\mathrm{NH}_{2}$ terminus of the anion. ${ }^{3}$ Femtosecond spectroscopy has been used to examine the hydration dynamics of various proteins by exciting tryptophan residues within the protein and measuring the solvent relaxation. ${ }^{4,5}$ These studies have provided an "outside looking in" view of the solvation structure of amino acids, viz., examining the solvent to characterize its interaction with the solute.

Soft X-ray spectroscopy has also been used extensively to characterize the electronic structure of amino acids and peptides. ${ }^{6-19}$ Near-edge X-ray absorption fine structure (NEXAFS), inner shell electron energy loss spectroscopy (ISEELS), and scanning transmission X-ray microscopy (STXM) have been

\footnotetext{
$\dagger$ University of California.

$\doteqdot$ Lawrence Berkeley National Laboratory.
}

employed in studies of spin-cast thin films of all 20 basic amino acids, ${ }^{14}$ amino acid monolayers adhered to metallic substrates, ${ }^{7,8}$ and both vapor ${ }^{17-19}$ and solid thin film samples of specific amino acids and their respective di- and tripeptides. ${ }^{6,15,16,20}$ These experiments have been supported by a variety of theoretical efforts. ${ }^{7,8,13,14,21}$

We have recently explored the effect of $\mathrm{pH}$ on the electronic structure of glycine in aqueous solution using NEXAFS spectroscopy. ${ }^{22}$ Several features in the nitrogen K-edge NEXAFS spectrum of aqueous glycine exhibited marked $\mathrm{pH}$ sensitivity. Comparison of these features to spectra of glycine absorbed on metal substrates ${ }^{7,8}$ and to theoretical calculations ${ }^{22}$ suggests that the nitrogen terminus of glycine is incompletely hydrated at high $\mathrm{pH}$ and exists in an "acceptor-only" position, wherein neither amine proton is involved in hydrogen bonding to the solvent. This new insight into the local hydration environment was unexpected, and demonstrated the sensitivity of NEXAFS spectroscopy to changes in the solvation environment of amino acids. In an attempt to further explore the local hydration environments of amino acids, we have measured and assigned the $\mathrm{pH}$ dependent NEXAFS spectra of the amino acids proline and lysine, and the dipeptide diglycine. The nitrogen terminus of proline is a secondary amine, unlike the more typical primary amine terminus of glycine. We have previously suggested that the spectral changes in the nitrogen K-edge spectrum of glycine are due to conformational changes of the molecule, specifically rotation of the amine about the $\mathrm{C}-\mathrm{N}$ bond. ${ }^{22}$ This rotation causes the amine protons to be sterically shielded from the surrounding solvent molecules. Proline, being a secondary amine, is incapable of this type of rotation, and, as 
such, should exhibit marked differences in the $\mathrm{pH}$ dependence of its nitrogen K-edge spectrum if the spectral changes are indeed engendered by conformational changes. Specifically, the sharp, $\mathrm{pH}$ dependent preedge $1 \mathrm{~s} \rightarrow \sigma^{*}{ }_{\mathrm{NH}}$ resonances observed in the NEXAFS spectrum of glycine in basic solution should be significantly broadened and diminished in intensity for proline.

The negatively charged carboxylate moiety of an anionic amino acid is in close proximity to its terminal amine. Not only does this nearby source of electron density stabilize the conformational rearrangement of the amino acid, it also acts as a strong orientational perturbation on nearby solvent molecules. Raman studies have demonstrated that the strength of this perturbation on the solvent molecules falls off steadily as the distance between the two moieties is increased. ${ }^{23}$ In an actual protein, the nitrogen and carbon termini can be separated by large distances, ${ }^{1}$ and it remains to be seen if the neighboring peptide bond provides sufficient electron density to support the acceptor-only conformation observed in the individual amino acid. Diglycine represents the simplest test of this effect. The separation between the nitrogen and carbon termini in diglycine is roughly twice that of glycine ( $\sim 5 \AA$ vs $\sim 2.5 \AA$, respectively). If the perturbation in local solvent structure caused by the carboxylate moiety is necessary for the existence of an acceptoronly species, then there should be no evidence of that configuration in the NEXAFS spectrum of anionic diglycine.

At neutral $\mathrm{pH}$, the amino acid lysine exists as a cation. Lysine's buytlamine side chain is a stronger base than its $\alpha$ amine, enabling zwitterionic lysine's $\alpha$ amine to potentially assume an acceptor-only configuration. Additionally, the intramolecular charge separation in lysine is roughly twice that of zwitterionic glycine ( $\sim 5.8 \AA$ vs $\sim 3.25 \AA$, respectively). This increased charge separation will enhance the disruption of the local hydrogen bond network, ${ }^{23}$ facilitating the formation of an acceptor-only $\alpha$ amine. Lysine's nitrogen K-edge spectrum will contain features from both $\alpha$ and side chain amines, and as such, should strongly resemble an average of the nitrogen K-edge spectra of glycine at neutral and basic $\mathrm{pH}$.

Here we report a comparative study of the nitrogen K-edge NEXAFS spectra of aqueous glycine, proline, and diglycine at neutral and basic $\mathrm{pH}$, and of aqueous zwitterionic lysine. The acidic solution $\mathrm{N}$ K-edge spectra are expected to behave identically to the zwitterion. ${ }^{22}$ To overcome the technical difficulties inherent in studying volatile liquids, and to avoid the problems associated with radiation damage to the sample, we have exploited the technology of liquid microjets originally developed by Faubel et al. ${ }^{24}$ and further developed by Wilson et al. ${ }^{25-28}$ Extensive density functional theory calculations were performed with a commercial software package ${ }^{29}$ to make spectral assignments and to analyze the effect of $\mathrm{pH}$ on the electronic structure of aqueous proline, diglycine, and lysine. To our knowledge, this is the first such study of these systems.

\section{Experimental and Computational Methods}

2.1. Samples. Glycine $\left(\mathrm{C}_{2} \mathrm{H}_{5} \mathrm{NO}_{2}\right)$, proline $\left(\mathrm{C}_{5} \mathrm{H}_{9} \mathrm{NO}_{2}\right)$, diglycine $\left(\mathrm{C}_{4} \mathrm{H}_{8} \mathrm{~N}_{2} \mathrm{O}_{3}\right)$, and lysine $\left(\mathrm{C}_{6} \mathrm{H}_{14} \mathrm{~N}_{2} \mathrm{O}_{2}\right)$ were obtained commercially from Fischer Scientific in the form of crystalline powders with a stated purity of better than $99 \%$. These were used without further purification. Initial amino acid solutions ( $10 \%$ by mass) were prepared with $18 \mathrm{M} \Omega$ water (Millipore). The solution $\mathrm{pH}$ was adjusted by adding reagent grade $\mathrm{NaOH}$ (Fischer) prior to dilution. These stock solutions were further diluted to $5 \%$ by mass $(\sim 0.6 \mathrm{M}$ glycine, $\sim 0.4 \mathrm{M}$ proline, or $\sim 0.3 \mathrm{M}$ lysine). Due to decreased solubility, diglycine was prepared directly as a $5 \%$ by mass solution $(\sim 0.4 \mathrm{M})$.
2.2. NEXAFS Spectroscopy of Aqueous Solutions. The $\mathrm{X}$-ray absorption spectra were recorded over the nitrogen K-edge $(380-430 \mathrm{eV})$ at Beamline 8.0.1 of the Advanced Light Source at Lawrence Berkeley National Laboratory in Berkeley, CA. A detailed description of the experimental apparatus has recently been given by Wilson et al. ${ }^{27}$ and the specifics of this work are identical with those previously reported. ${ }^{22}$ The spectra presented in this work have been normalized to the excitation intensity. The background is then subtracted to produce a level baseline and the spectra are area normalized to $415 \mathrm{eV}$.

2.3. Computational Methods. To aid in the interpretation of the spectra collected in these experiments, the NEXAFS spectra of hydrated zwitterionic and anionic glycine and proline were calculated by using density functional theory and transitionpotential methods. The StoBe DeMon 2.029 software package is a commercially available implementation of these approaches, and was used for all energetic and spectral calculations presented in this work. The basis functions and correlation functional used have been previously described in detail. ${ }^{22}$ Initial geometries were created via a molecular mechanics conformation search conducted on the amino acid with several solvent water molecules, using a commercial software package (MacroModel). Seven and ten water molecules were included for glycine and for all other species, respectively. With the exception of diglycine, the geometries of several low-energy candidate clusters were then optimized by using the StoBe DFT package. In all cases, only minor conformational changes were found to be within $\mathrm{kT}$ of the global minimum structure. Those clusters with qualitatively different hydrogen bonding networks were found to be of much higher energy, and were not included in the calculated XAS spectra. For clarity, the XAS spectra of the lowest energy cluster are presented in this work. Conformational changes within kT of the lowest energy structure were not found to differ qualitatively from the calculated spectra presented. These minor shifts in position and intensity are masked by the Lorentzian convolution used to produce the spectra presented in this work. In the case of diglycine, 10 water molecules are insufficient to completely hydrate the dipeptide. There is a software limit of 11 separate molecules in the MM package used for conformational searches, limiting the size of the available solvation shell. Therefore, the isolated molecule was used for geometry optimization, and water molecules were added to create a cluster wherein every possible hydrogen bond was formed. While this system should not be considered an accurate model of solvated diglycine, it is adequate to determine the effect of hydrogen bonding on the nitrogen K-edge spectrum.

The basis set changes required to calculate the NEXAFS spectrum of each conformer have been described elsewhere. ${ }^{22}$ The width of each transition was held constant at $1 \mathrm{eV}$ for transitions below $402 \mathrm{eV}, 2.5 \mathrm{eV}$ for transitions above $403 \mathrm{eV}$, and linearly interpolated between 402 and $403 \mathrm{eV}$. These values were chosen to reflect the widths observed in previous experimental spectra, as well as to reasonably reproduce the spectra measured in this work. Transition assignments were obtained by examining the spatial extent of the molecular orbital of the final state, comparison of relative oscillator strengths and positions, and by visual examination of the final state by plotting the molecular orbital using the Molekel 4.3 graphics package. ${ }^{30}$ Varying the exchange/correlation functionals and basis sets used was found to change the absolute value and position of the calculated transitions, but the relative spacing and intensities were well preserved, requiring only a constant energetic offset to provide consistent agreement with the experimental spectra. 
a)

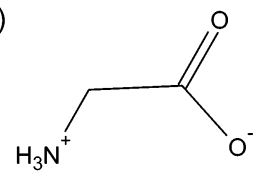

c)

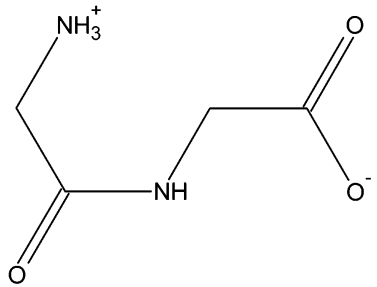

b)

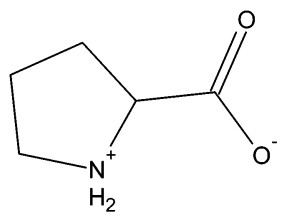

d)

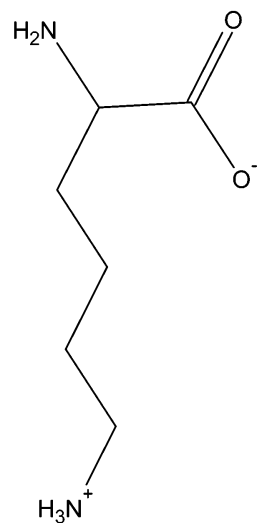

Figure 1. Structural representations of zwitterionic glycine (a), proline (b), diglycine (c), and lysine (d). The $\alpha$ amines of glycine and lysine are free to rotate about the $\mathrm{C}-\mathrm{N}$ bond, whereas proline contains a secondary amine that is locked into position. The terminal amine of diglycine is separated by twice the distance from the carboxlate moiety relative to glycine ( $4.9 \AA$ vs $2.45 \AA$, respectively).

\section{Results and Discussion}

Amino acids can exist in three distinct charge states, dependent upon the solution $\mathrm{pH}$. For this work, we have focused on the zwitterionic and anionic forms of glycine, proline, and diglycine, and the zwitterionic form of lysine. The systematic comparison of the nitrogen K-edge spectra of these species provides a complete picture of the hydration environment of the nitrogen terminus of amino acids and polypeptides.

3.1. Comparison of Glycine and Proline. As previously reported in the complete $\mathrm{pH}$ characterization of glycine, ${ }^{22}$ the nitrogen K-edge spectra of glycine are identical for the acidic and zwitterionic species. This is not surprising, as the nitrogen termini of both species are identical. As shown in Figure 1a, the nitrogen terminus of glycine is a primary amine, and both the $-\mathrm{NH}_{3}{ }^{+}$and $-\mathrm{NH}_{2}$ subgroups are free to rotate about the $\mathrm{C}-\mathrm{N}$ bond. In contrast, proline's cyclic side chain transforms the $\alpha$ nitrogen into a secondary amine (Figure 1b), preventing any such rotation about the $\alpha \mathrm{C}-\mathrm{N}$ bond. The remaining $\mathrm{NH}$ bonds are therefore fixed relative to the carboxylic acid moiety, although at high $\mathrm{pH}$ values, the extra proton is free to exist either on the same or on the opposite side of the cyclic side chain as the carboxyl moiety. Both conformations are energetically similar, and at room temperature, both are expected for the isolated molecule. For the NEXAFS calculations presented in this work, we have used the slightly more stable cis conformer. Of the two conformers, the cis is more likely to exhibit steric shielding by the carboxyl group. Thus, if the cis conformer of proline exhibits the same decrease in preedge intensity upon hydration as glycine, we can be confident that the trans conformer will as well.

Figure 2 depicts the TEY NEXAFS spectra of zwitterionic (black) and anionic (gray) glycine (Figure 2a) and proline (Figure 2b). All four spectra can be described as exhibiting a strong main edge absorption near 406.5 or $405 \mathrm{eV}$ for the zwitterionic and anionic solutions, respectively. This supports the previous conclusion ${ }^{22}$ that this spectral shift is due to the difference in ionization potential between the $-\mathrm{NH}_{3}{ }^{+}$moiety

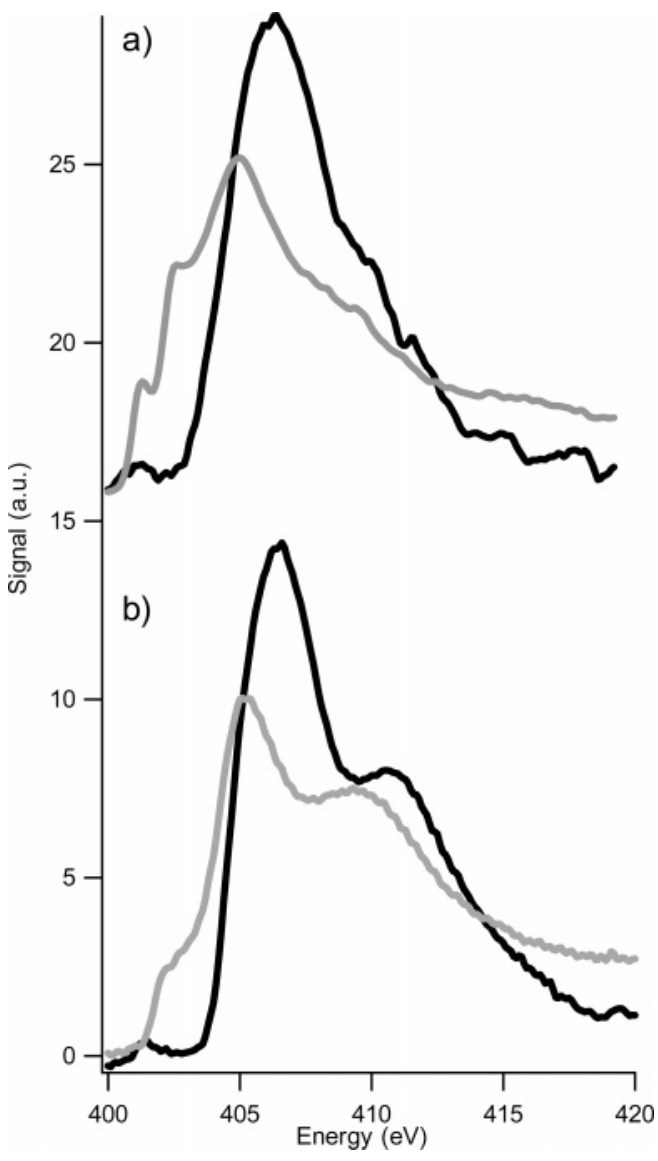

Figure 2. Area normalized TEY NEXAFS N K-edge spectra of (a) glycine at $\mathrm{pH} 6.1$ (black) and $\mathrm{pH} 11.8$ (gray) and (b) proline at $\mathrm{pH} 6.3$ (black) and $\mathrm{pH} 12.2$ (gray). Both species show a $\sim 1.3 \mathrm{eV}$ red shift at high $\mathrm{pH}$. The preedge resonances of proline have been reduced to a broad shoulder, and proline's postedge intensity is increased compared to that of glycine.

in the zwitterion and the $-\mathrm{NH}_{2}$ moeity in the anion. Additionally, all four species exhibit a broad resonance approximately $3 \mathrm{eV}$ to the blue of the main edge, although this feature is much more pronounced in proline than in glycine.

In anionic form, both glycine and proline exhibit additional spectral features to the red of the main edge. In glycine, the preedge resonance at $401.3 \mathrm{eV}$ is assigned to a $1 \mathrm{~s} \rightarrow \sigma^{*} \mathrm{NH}_{2}$ transition. The final state of this resonance is localized along both of the amine terminus' $\mathrm{NH}$ bonds, in a manner analogous to the $3 \mathrm{a}_{1}$ orbital of ammonia. ${ }^{31,32} \mathrm{We}$ have assigned the second preedge at $402.6 \mathrm{eV}$ to a pair of near degenerate $1 \mathrm{~s} \rightarrow \sigma^{*}{ }_{\mathrm{NH}}$ transitions. These transitions can be distinguished from the $1 \mathrm{~s}$ $\rightarrow \sigma^{*} \mathrm{NH}_{2}$ transition as being localized along one-half of the terminal amine group, similar to the 2e orbitals of ammonia. ${ }^{31,32}$ These well-resolved features are not present in the TEY NEXAFS of anionic proline, and the only observed preedge intensity is a broad shoulder at $402.2 \mathrm{eV}$. To identify this shoulder resonance, we have compared the calculated NEXAFS spectra of the isolated and hydrated amino acids.

NEXAFS spectroscopy probes the unoccupied molecular orbitals and Rydberg states of the excited molecule. ${ }^{33}$ Because of the large spatial extent of these final states, the position and intensity of NEXAFS transitions are very sensitive to changes in the local environment of the atom being probed. To examine these effects, we have calculated the nitrogen K-edge NEXAFS spectra of isolated glycine and various hydrated glycine clusters. As shown in Figure 3, isolated forms of both the zwitterion and anion show sharp preedge transitions (gray curves in Figures 


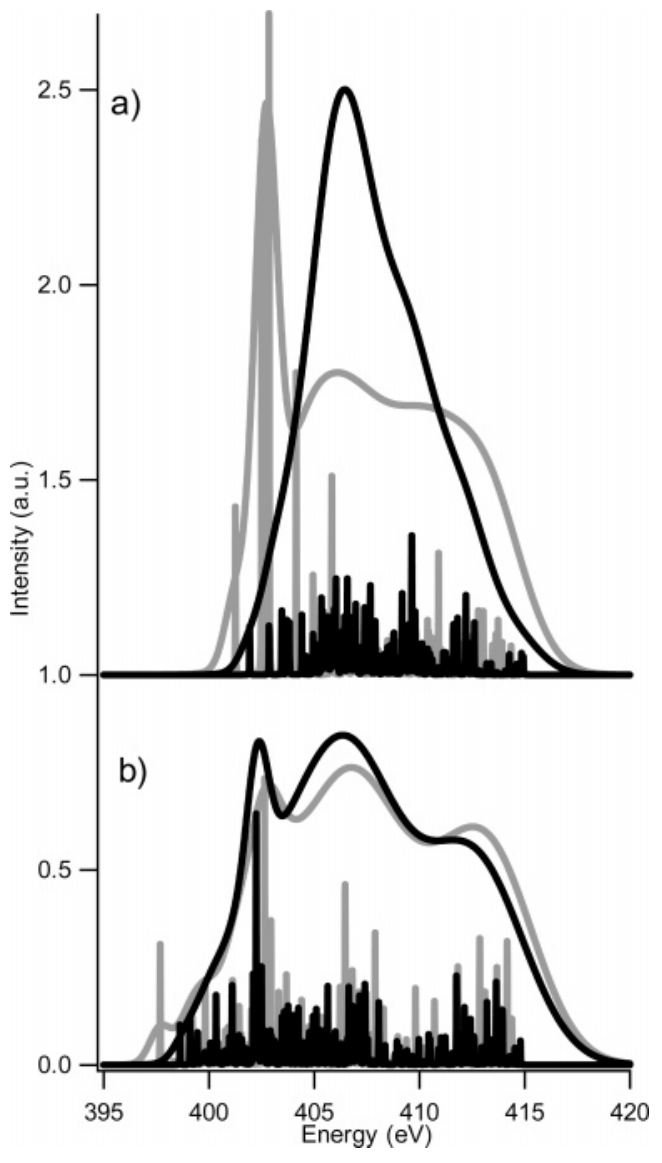

Figure 3. Calculated glycine N K-edge NEXAFS spectra with (black) and without (gray) water at neutral (a) and basic (b) pH. In both cases, complete hydration of the terminal amine reduces the number and intensity of preedge transitions.

$3 \mathrm{a}$ and $3 \mathrm{~b}$, respectively). These resonances can be unambiguously assigned to $1 \mathrm{~s} \rightarrow \sigma^{*}{ }_{\mathrm{NH}}$ transitions. The smaller preedge resonances predicted near 401 and $398 \mathrm{eV}$ for the zwitterion and anion, respectively, contain character from multiple $\mathrm{NH}$ bonds. The strong resonances near $403 \mathrm{eV}$ comprise neardegenerate resonances localized along a $\mathrm{NH}$ single bond. When placed in a hydrated cluster, the zwitterion donates all three protons in hydrogen bonds to the surrounding solvent molecules. The transitions predicted in Figure 3 are dipole allowed, and the formation of a hydrogen bond and subsequent addition of a second nearby nodal plane reduces the p-character of the final state, dramatically reducing in transition intensity. The resulting hydrated spectrum is shown as the black curve in Figure 3a.

Several initial geometries were generated for hydrated clusters of anionic glycine. In all cases, the $\alpha$ amine moiety was found in an acceptor-only state after energetically optimizing the cluster geometry, and the resulting clusters differed only in the position of the solvent water molecules. The large electron density of the nearby carboxyl group perturbs both the nearby solvent molecules and the $\alpha$ amine, reorienting the solvent molecules and promoting a rotation about the $\mathrm{CN}$ amine bond. In this environment, there are no sterically accessible solvent molecules to accept a hydrogen bond from the $\alpha$ amine. The resulting hydrated anion spectrum is shown as the black curve in Figure 3b. The strong $1 \mathrm{~s} \rightarrow \sigma^{*}{ }_{\mathrm{NH}}$ resonance near $403 \mathrm{eV}$ remains, indicating that the antibonding orbital localized along one of the $\mathrm{NH}$ bonds is relatively unperturbed. Meanwhile, the smaller resonance near $398 \mathrm{eV}$ is diminished in intensity, suggesting that the collective $\mathrm{NH}_{2} \sigma^{*}{ }_{\mathrm{NH}}$ has been perturbed by the surrounding solvent.
These assignments are in agreement with the polarizationdependent NEXAFS spectra of glycine chemisorbed on $\mathrm{Cu} .{ }^{7,8}$ The surface packing structure of glycine on $\mathrm{Cu}$ [110] is asymmetric with respect to the terminal nitrogen, with one $\mathrm{NH}$ bond donating a hydrogen bond to the carboxyl group of an adjacent glycine and the other $\mathrm{NH}$ group remaining unbound. When the excitation polarization is aligned perpendicular to the plane of the $\mathrm{NH}_{2}$ group, both preedge resonances are observed. When aligned with the NH bond involved in hydrogen bonding, neither resonance is observed. When aligned with the free $\mathrm{NH}$ bond, only the higher energy $1 \mathrm{~s} \rightarrow \sigma^{*}{ }_{\mathrm{NH}}$ resonance is observed. The $1 \mathrm{~s} \rightarrow \sigma^{*} \mathrm{NH}_{2}$ transition is no longer allowed due to the broken symmetry caused by formation of a hydrogen bond by the other $\mathrm{NH}$ bond. When compared to the experimental TEY NEXAFS spectrum of aqueous glycine, we find that the nitrogen terminus is completely hydrated at moderate and low $\mathrm{pH}$, and in an acceptor-only configuration at high $\mathrm{pH} .^{22}$ This is somewhat surprising, given that aqueous glycine molecules are assumed to have a complete solvation shell surrounding each glycine at the concentrations studied.

Unlike the other naturally occurring amino acids, the nitrogen terminus of proline forms a secondary amine with the $\alpha$ carbon and the $\delta$ carbon of the side chain propyl group. It is not possible to directly determine whether the acceptor-only conformation observed in anionic glycine is due to the reorientation of the solvation shell or from conformational changes in the glycine anion itself. Proline, being a secondary amine, is incapable of the conformational changes suggested for glycine. As such, if the acceptor only conformation observed in anionic glycine is reliant only on reorienting the solvent shell then similar reorientation should occur for anionic proline. The spectrum of anionic proline (Figure $2 b$, gray) lacks the sharp preedge features seen in anionic glycine (Figure 2a, gray), although there is a broad shoulder resonance at $402.2 \mathrm{eV}$. If this shoulder resonance is due to a $1 \mathrm{~s} \rightarrow \sigma^{*}{ }_{\mathrm{NH}}$ transition, then it has been considerably broadened in comparison to the analogous glycine transition. This shoulder is therefore either due to a different set of transitions or the $1 \mathrm{~s} \rightarrow \sigma^{*} \mathrm{NH}$ transition has experienced significant environmental broadening, suggesting a variety of solvation environments in anionic proline. Comparison to calculation can distinguish between these two possibilities.

The calculated spectra of isolated and hydrated proline are depicted in Figure 4 by gray and black curves, respectively. The zwitterionic spectra are shown in Figure 4a, while those of the anion are shown in Figure 4b. For both isolated species (gray curves in Figure 4), strong resonances corresponding to $1 \mathrm{~s} \rightarrow$ $\sigma^{*} \mathrm{NH}$ transitions are predicted to occur prior to the main nitrogen step edge. For the isolated zwitterion, these resonances are energetically near enough to the main edge to make them spectroscopically unresolvable, and will produce, at most, a shoulder on the main edge. For the isolated anion, this $1 \mathrm{~s} \rightarrow$ $\sigma^{*} \mathrm{NH}$ transition predicted near $401 \mathrm{eV}$ is both energetically resolvable and predicted to be similar in intensity to the main edge itself (Figure 4b, gray). These resonances are either absent or significantly reduced in intensity in the calculated spectra of hydrated proline clusters (black curves in Figure 4). The very intense and well-resolved preedge feature predicted in Figure $4 \mathrm{~b}$ is not observed in the experimental spectrum of anionic proline (Figure 2b, gray). Additionally, we have assigned the preedge shoulder resonance at $402.2 \mathrm{eV}$ to a combination of $1 \mathrm{~s}$ $\rightarrow \sigma^{*} \mathrm{CN}, \pi^{*} \mathrm{CC}$, and Rydberg state transitions. From this assignment we conclude that anionic proline is completely hydrated, in agreement with the physical interpretation previously described. ${ }^{22}$ 


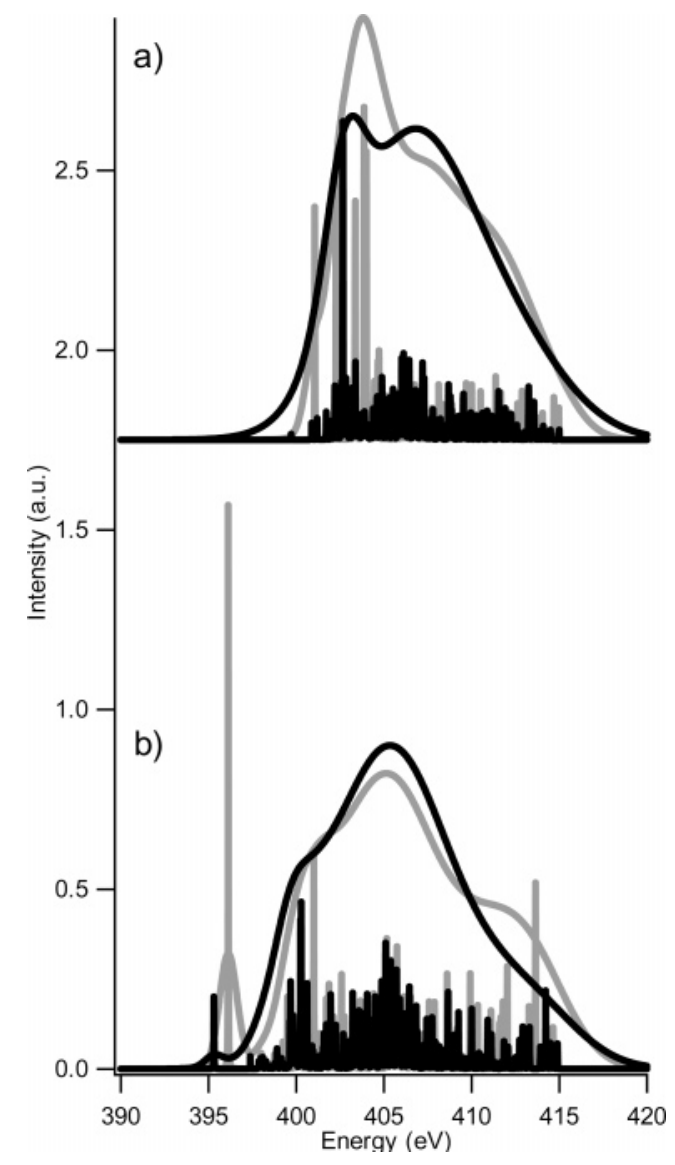

Figure 4. Calculated proline N K-edge NEXAFS spectra with (black) and without (gray) water at neutral (a) and basic (b) $\mathrm{pH}$. An additional strong $1 \mathrm{~s} \rightarrow \sigma^{*}{ }_{\mathrm{NH}}$ resonance $8 \mathrm{eV}$ red of the main edge is predicted at high $\mathrm{pH}$ for the unhydrated molecule. The preedge shoulder appears to be insensitive to hydration, and is assigned to $1 \mathrm{~s} \rightarrow \sigma^{*} \mathrm{CN}, \pi^{*} \mathrm{CC}$, and Rydberg states. These assignments suggest that proline is completely hydrated at all $\mathrm{pH}$ values.

The additional spectral features in Figure $2 b$ are similar to those found in Figure 2a. The main-edge at $406.5 \mathrm{eV}$ (zwitterion) and at $405.1 \mathrm{eV}$ (anion) arises from contributions from the nitrogen step edge and $1 \mathrm{~s} \rightarrow \sigma^{*} \mathrm{CC}, \sigma^{*} \mathrm{CN}, \pi^{*} \mathrm{CN}$, and $\pi^{*} \mathrm{CC}$ resonances. The broad postedge resonances centered at 410.6 (zwitterion) and $409.4 \mathrm{eV}$ (anion) can be assigned to transitions to $1 \mathrm{~s} \rightarrow \pi^{*} \mathrm{CO}_{2}$ and $\sigma^{*} \mathrm{NH}$ resonances. There is also a large collection of resonances to various states localized on the side chain. These side chain resonances significantly increase the density of states in this region compared to the case of glycine, and account for the observed increase in intensity in this region for proline.

3.2. Comparison of Glycine and Diglycine. Figure 5 shows a comparison of the $\mathrm{pH}$ dependent glycine (Figure 5a) N K-edge spectra with those of diglycine (Figure $5 \mathrm{~b}$ ). Diglycine contains a peptide bond connecting its glycine residues, and the resulting $1 \mathrm{~s} \rightarrow \pi^{*}$ CON resonance occurs at $401.3 \mathrm{eV} .{ }^{6}$ With the exception of this new peak, the nitrogen spectra of both zwitterionic species (Figure 5, black) are quite similar. Both exhibit strong main edges at $406.3 \mathrm{eV}$, and a broad postedge shoulder. This broad shoulder is shifted further to the blue $(\sim 411.4 \mathrm{eV})$ in the diglycine spectrum than in glycine $(\sim 409 \mathrm{eV})$. This high-energy shoulder resonance in diglycine is analogous to the shoulder resonances of glycine and proline. The observed blue shift in this shoulder resonance upon formation of the peptide bond is not surprising, as diglycine contains a greater number of bonds than glycine, as well as an additional hydrogen bond location, thereby increasing the density of states in this energy region.

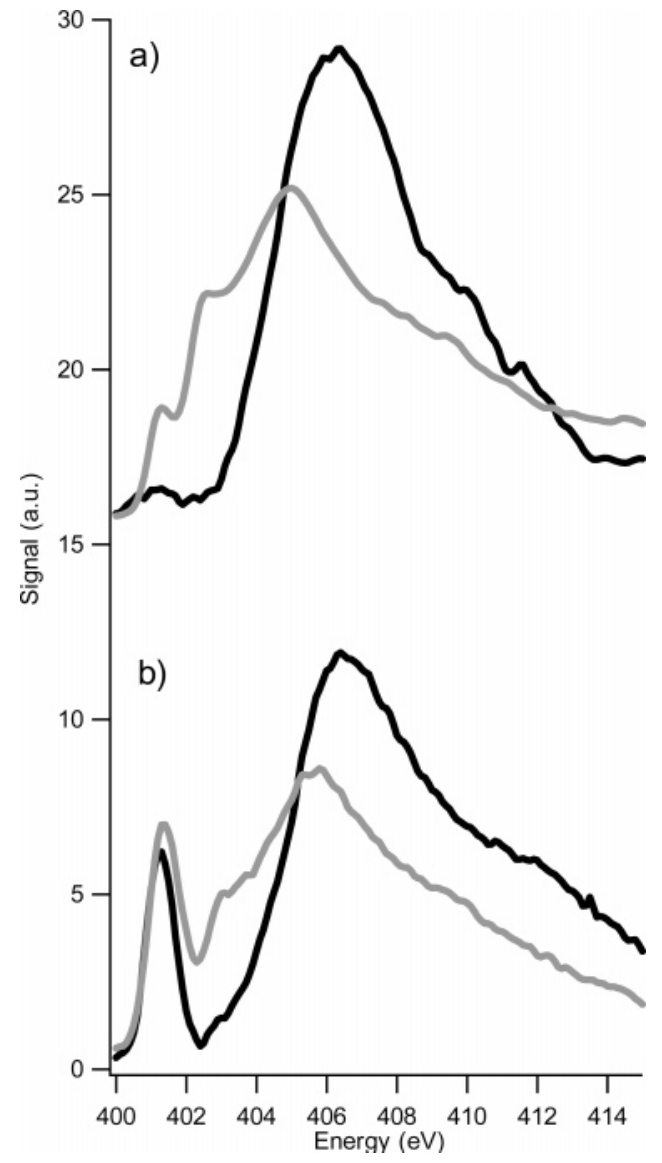

Figure 5. Area normalized TEY NEXAFS N K-edge spectra of (a) glycine at pH 6.1 (black) and pH 11.8 (gray) and (b) diglycine at neutral $\mathrm{pH}$ (black) and basic (gray) $\mathrm{pH}$. Anionic diglycine lacks the wellresolved $1 \mathrm{~s} \rightarrow \sigma^{*}{ }_{\mathrm{NH}}$ resonance observed in anionic glycine. There is a broad shoulder resonance at $403 \mathrm{eV}$, and the $1 \mathrm{~s} \rightarrow \pi^{*} \mathrm{CN}$ resonance at $401 \mathrm{eV}$ is broadened, indicating that an acceptor-only species is possible.

At high $\mathrm{pH}$, three spectral changes are observed in the nitrogen K-edge spectrum of anionic diglycine. First, the $1 \mathrm{~s} \rightarrow$ $\pi^{*}$ CON is slightly broadened compared to the same resonance in the zwitterionic spectrum. Second, the main edge is red shifted by $1.2 \mathrm{eV}$, similar to the shift observed in both glycine and proline. Finally, a broad low-energy shoulder resonance is observed at $403.5 \mathrm{eV}$, at an energy similar to the $1 \mathrm{~s} \rightarrow \sigma^{*} \mathrm{NH}$ preedge transition of anionic glycine. These spectral differences between the zwitterion and anion are very similar to those observed for glycine, although the $1 \mathrm{~s} \rightarrow \sigma^{*} \mathrm{NH}_{\mathrm{N}}$ transitions appear to be broadened significantly for anionic diglycine.

The gas and solid phase inner-shell spectra of diglycine have been previously reported. ${ }^{6,20} \mathrm{We}$ find that the aqueous solution measurements are in good agreement with the previously assigned spectra. As discussed in our previous study on glycine ${ }^{22}$ the vapor phase spectrum of an amino acid strongly resembles that of the aqueous system at high $\mathrm{pH}$ while the solid resembles that of the zwitterionic solution. These similarities are preserved in the case of diglycine, with the neutral solution strongly resembling the solid-phase spectrum, and the spectrum of the basic solution resembling that of the gas phase. The $1 \mathrm{~s}$ $\rightarrow \pi^{*}$ CON transition is broader in the gas-phase spectrum than in the basic solution spectrum. This is due in part to the decreased spectral resolution of the gas-phase spectrum, ${ }^{6}$ although the $1 \mathrm{~s} \rightarrow \sigma^{*} \mathrm{NH}$ transitions appear to be more pronounced in the gas phase.

The calculated NEXAFS spectra of the hydrated (black) and isolated (gray) diglycine zwitterion and anion are presented in 


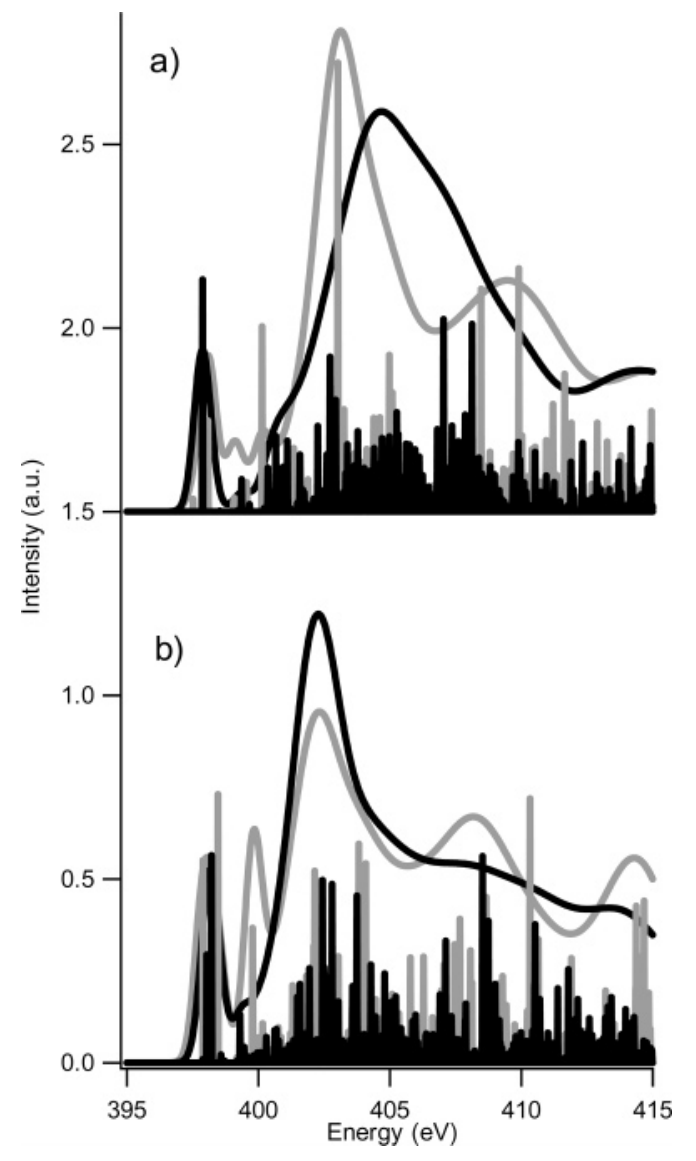

Figure 6. Calculated diglycine N K-edge NEXAFS spectra with (black) and without (gray) water at neutral (a) and basic (b) $\mathrm{pH}$. The only peak predicted at $403 \mathrm{eV}$ for the anion is the $1 \mathrm{~s} \rightarrow \sigma^{*}{ }_{\mathrm{NH}}$ resonance, although significant broadening would be necessary to create the preedge shoulder observed in the experimental spectrum.

Figure 6, parts a and b, respectively. As with glycine (Figure 3 ) and proline (Figure 4), there is a general qualitative agreement between the experimental and calculated spectra. In the case of anionic diglycine, the $1 \mathrm{~s} \rightarrow \sigma^{*} \mathrm{NH}_{2}$ transition is predicted to be very close to the $1 \mathrm{~s} \rightarrow \pi^{*}$ CON transition. This overlap is responsible for the apparent broadening of the $1 \mathrm{~s} \rightarrow \pi^{*} \mathrm{CON}$ transition observed in the experimental spectrum (Figure 5b). Additionally, the only strong transitions predicted between the $1 \mathrm{~s} \rightarrow \pi^{*} \mathrm{CON}$ and the main edge arise from $1 \mathrm{~s} \rightarrow \sigma^{*}{ }_{\mathrm{NH}}$ transitions. This indicates that, as with glycine, the nitrogen terminus of diglycine is in an acceptor only configuration at high $\mathrm{pH}$. It is important to note, however, that the diglycine $1 \mathrm{~s} \rightarrow \sigma^{*} \mathrm{NH}$ transitions are broadened in comparison to those of glycine. This broadening could be due to additional contributions from weaker transitions in this energy range or from inhomogeneous broadening that does not occur with glycine. The peptide bond connecting diglycine's two residues is much lower in local electron density than its carboxylate terminus. This decreased electron density will likely create a smaller perturbation on the local hydrogen bonding environment surrounding the nitrogen terminus. As such, it is possible that the nitrogen terminus of diglycine exists in a greater variety of hydration environments, and the acceptor-only configuration comprises a smaller fraction of these configurations than it does in the case of glycine.

3.3. Comparison of Glycine and Lysine. As a final verification of the previous interpretation given, ${ }^{22}$ the $\mathrm{pH}$ dependent TEY NEXAFS spectra of glycine are compared to the zwitterionic spectra of aqueous lysine in Figure 7. With its basic butylammonium side chain, lysine has an isoelectric point

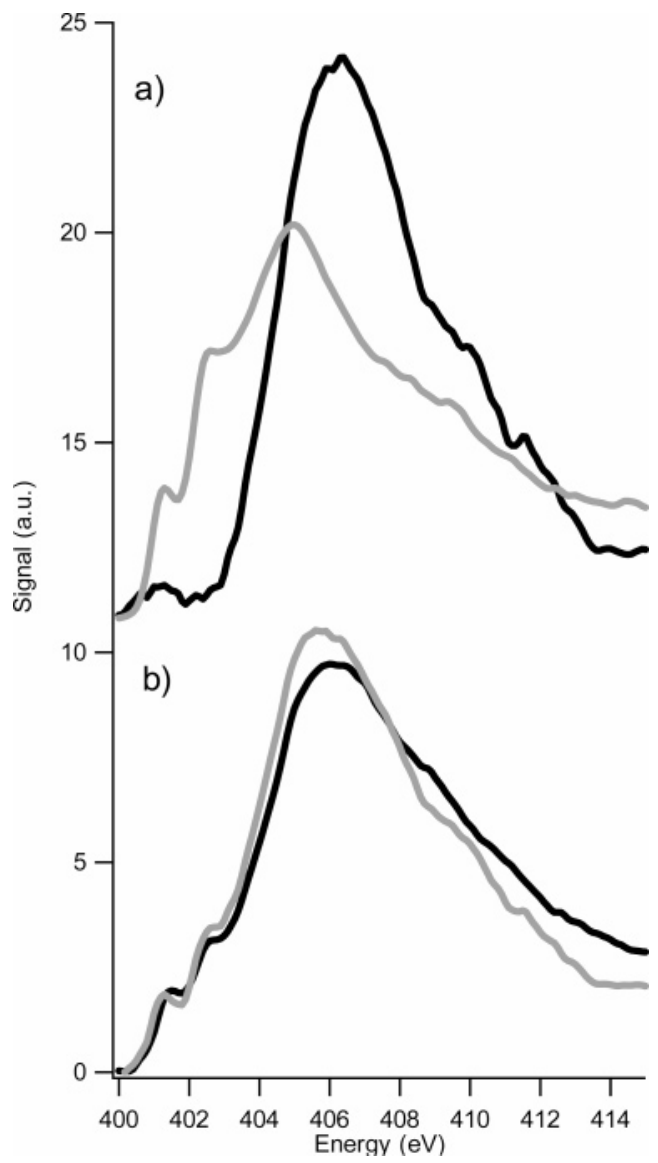

Figure 7. Area normalized TEY NEXAFS N K-edge spectra of (a) glycine at pH 6.1 (black) and pH 11.8 (gray) and (b) lysine at pI (black) and the average sum of the curves in part a (gray). Lysine at $\mathrm{pI}(\mathrm{pH}$ 10) exhibits features of both $-\mathrm{NH}_{2}$ and $-\mathrm{NH}_{3}{ }^{+}$moieties, as expected from its zwitterionic structure. The $\alpha$ amine is present in an acceptoronly configuration, similar to that of anionic glycine.

(pI) of $\sim 10$, and as a zwitterion its $\alpha$ amine is deprotonated. ${ }^{1}$ Additionally, the long butyl side chain increases the intramolecular charge separation between the ammonium and carboxylate moieties. Normally, the close proximity of these two moieties diminishes disruption in the local hydrogen bond structure. $^{23}$ In this case, the $\alpha$ amine is left in an ideal environment to take on the acceptor-only configuration observed in anionic glycine. The ammonium terminus of lysine's butylamine side chain is expected to be completely hydrated, in a manner similar to the $\alpha$ amine of zwitterionic glycine. As such, the predicted $\mathrm{N}$ K-edge spectrum of zwitterionic lysine is expected to contain features similar to those of both anionic and zwitterionic glycine. As seen in Figure 7b, there is a strong similarity between the TEY NEXAFS of zwitterionic lysine (black) and the average spectrum of zwitterionic and anionic glycine (gray). These small spectral differences can easily be assigned to the presence of the butyl side chain in lysine.

\section{Summary}

The nitrogen K-edge spectra of aqueous proline and diglycine solutions have been measured by total electron yield NEXAFS at neutral and high $\mathrm{pH}$, as has the NEXAFS spectrum of zwitterionic lysine in aqueous solution. Comparison with the $\mathrm{pH}$ dependent NEXAFS glycine spectra, as well as calculated spectra of isolated amino acids and hydrated amino acid clusters, has allowed us to completely assign all observed spectral features. From the sharp preedge resonances at 401.3 and 402.6 $\mathrm{eV}$ in the spectrum of anionic glycine, we conclude that the 
nitrogen terminus is in an acceptor-only configuration at high $\mathrm{pH}$. In comparison, the analogous $1 \mathrm{~s} \rightarrow \sigma^{*} \mathrm{NH}$ preedge transition is absent in the NEXAFS spectrum of anionic proline. This implies that the acceptor-only conformation observed in anionic glycine is due primarily to the steric shielding from solvent molecules induced by rotation of the amine moiety about the glycine $\mathrm{CN}$ bond. As this rotation is impossible in the secondary amine proline, we conclude that the perturbation in solvent structure caused by the nearby carboxylate moiety is necessary but insufficient to produce the acceptor-only species observed in anionic glycine.

It is possible that the sodium hydroxide used to adjust the $\mathrm{pH}$ of the solutions used in this study acts as a perturbation to the local hydration environment of the amino acids and dipeptide studied. However, the nitrogen K-edge spectrum of aqueous lysine also shows evidence of having an $\alpha$ amine in an acceptoronly configuration. It was not necessary to adjust the $\mathrm{pH}$ of the lysine solutions, indicating that the acceptor-only configurations observed in this study are not the result of the addition of the sodium hydroxide used in this study.

The nitrogen K-edge spectrum of diglycine is distinguished from that of glycine by the appearance of a $1 \mathrm{~s} \rightarrow \pi^{*} \mathrm{CON}$ resonance that can be observed at $401.3 \mathrm{eV}$. Additionally, the $1 \mathrm{~s} \rightarrow \sigma^{*} \mathrm{NH}$ preedge transitions in anionic diglycine are significantly broader than those observed in anionic glycine. This indicates that the nitrogen terminus of diglycine is capable of existing in an acceptor-only configuration, although it also appears to exist in a variety of hydration environments. From this we conclude that the peptide bond in diglycine provides sufficient electron density to support the acceptor-only observed in the individual amino acid. On the basis of these findings, the nitrogen terminus of most proteins should be capable of existing in an acceptor-only configuration at high $\mathrm{pH}$.

Acknowledgment. This research was supported by the Chemical Sciences, Geosciences and Biosciences Division, Office of Basic Energy Sciences, U.S. Department of Energy. C.D.C. is supported by the Advanced Light Source Doctoral Fellowship in Residence and the Department of DefenseNational Defense Science and Engineering Graduate Fellowship. The Advanced Light Source is supported by the Director, Office of Science, Office of Basic Energy Sciences, Materials Sciences Division, of the U.S. Department of Energy under contract no. DE-AC03-76SF00098 at Lawrence Berkeley National Laboratory. We warmly thank the Advanced Light Source support staff, especially J. Denlinger, M. Gilles, B.S. Rude, and D. Shuh, for their advice and support. B.M.M. would like to thank K. Hermann for invaluable advice and assistance with the StoBe 2.0 software package.

\section{References and Notes}

(1) Stryer, L. Biochemistry, 4th ed.; W. H. Freeman: New York, 1995.

(2) Lauterwein, J.; Gerothanassis, I. P.; Hunston, R. N.; Schumacher, M. J. Phys. Chem. 1991, 95, 3804.

(3) Troganis, A. N.; Tsanaktsidis, C.; Gerothanassis, I. P. J. Magn. Reson. 2003, 164, 294.
(4) Pal, S. K.; Peon, J.; Bagchi, B.; Zewail, A. H. J. Phys. Chem. B 2002, 106, 12376.

(5) Pal, S. K.; Peon, J.; Zewail, A. H. Proce. Natl. Acad. Sci. U.S.A. 2002, 99, 1763.

(6) Gordon, M. L.; Cooper, G.; Morin, C.; Araki, T.; Turci, C. C.; Kaznatcheev, K.; Hitchcock, A. P. J. Phys. Chem. A 2003, 107, 6144.

(7) Hasselstrom, J.; Karis, O.; Weinelt, M.; Wassdahl, N.; Nilsson, A.; Nyberg, M.; Pettersson, L. G. M.; Samant, M. G.; Stohr, J. Surf. Sci. 1998, 407, 221.

(8) Nyberg, M.; Hasselstrom, J.; Karis, O.; Wassdahl, N.; Weinelt, M.; Nilsson, A.; Pettersson, L. G. M. J. Chem. Phys. 2000, 112, 5420.

(9) Zubavichus, Y.; Zharnikov, M.; Schaporenko, A.; Grunze, M. J. Electron Spectrosc. Relat. Phenom. 2004, 134, 25.

(10) Zubavichus, Y.; Zharnikov, M.; Yang, Y. J.; Fuchs, O.; Heske, C.; Umbach, E.; Tzvetkov, G.; Netzer, F. P.; Grunze, M. J. Phys. Chem. B 2005, 109, 884 .

(11) Zubavichus, Y.; Zharnikov, M.; Shaporenko, A.; Fuchs, O.; Weinhardt, L.; Heske, C.; Umbach, E.; Denlinger, J. D.; Grunze, M. J. Phys. Chem. A 2004, 108, 4557.

(12) Zubavichus, Y.; Fuchs, O.; Weinhardt, L.; Heske, C.; Umbach, E.; Denlinger, J. D.; Grunze, M. Radiat. Res. 2004, 161, 346.

(13) Yang, L.; Plashkevych, O.; Vahtras, O.; Carravetta, V.; Agren, H. J. Synchrotron Radiat. 1999, 6, 708 .

(14) Kaznacheyev, K.; Osanna, A.; Jacobsen, C.; Plashkevych, O.; Vahtras, O.; Agren, H. A.; Carravetta, V.; Hitchcock, A. P. J. Phys. Chem. A 2002, 106, 3153.

(15) Tanaka, M.; Nakagawa, K.; Koketsu, T.; Agui, A.; Yokoya, A. J. Synchrotron Radiat. 2001, 8, 1009.

(16) Boese, J.; Osanna, A.; Jacobsen, C.; Kirz, J. J. Electron Spectrosc. Relat. Phenom. 1997, 85, 9.

(17) Cannington, P. H.; Ham, N. S. J. Electron Spectrosc. Relat. Phenom. 1983, 32, 139.

(18) Bomben, K. D.; Dev, S. B. Anal. Chem. 1988, 60, 1393.

(19) Richer, G.; Sandorfy, C.; Nascimento, M. A. C. J. Electron Spectrosc. Relat. Phenom. 1984, 34, 327.

(20) Cooper, G.; Gordon, M.; Tulumello, D.; Turci, C.; Kaznatcheev, K.; Hitchcock, A. R. J. Electron Spectrosc. Relat. Phenom. 2004, 137-40, 795.

(21) Carravetta, V.; Plashkevych, O.; Agren, H. J. Chem. Phys. 1998, $109,1456$.

(22) Messer, B. M.; Cappa, C. D.; Smith, J. D.; Wilson, K. R.; Gilles, M. K.; Cohen, R. C.; Saykally, R. J. J. Phys. Chem. B 2005, 109, 5375

(23) Kitano, H.; Takaha, K.; Gemmei-Ide, M. J. Colloid Interface Sci. 2005, 283, 452 .

(24) Faubel, M.; Schlemmer, S.; Toennies, J. P. Z. Phys. D: At. Mol. Clusters 1988, 10, 269.

(25) Wilson, K. R.; Cavalleri, M.; Rude, B. S.; Schaller, R. D.; Nilsson, A.; Pettersson, L. G. M.; Goldman, N.; Catalano, T.; Bozek, J. D.; Saykally, R. J. J. Phys.: Condens. Matter 2002, 14, L221.

(26) Wilson, K. R.; Rude, B. S.; Catalano, T.; Schaller, R. D.; Tobin, J. G.; Co, D. T.; Saykally, R. J. J. Phys. Chem. B 2001, 105, 3346.

(27) Wilson, K. R.; Rude, B. S.; Smith, J.; Cappa, C.; Co, D. T.; Schaller, R. D.; Larsson, M.; Catalano, T.; Saykally, R. J. Rev. Sci. Instrum. 2004, 75,725 .

(28) Wilson, K. R.; Schaller, R. D.; Co, D. T.; Saykally, R. J.; Rude, B. S.; Catalano, T.; Bozek, J. D. J. Chem. Phys. 2002, 117, 7738.

(29) Hermann, K.; Pettersson, L. G. M.; Casida, M. E.; Daul, C.; Goursot, A.; Koester, A.; Proynov, E.; St-Amant, A.; Salahub, D. R.; Carravetta, V.; Duarte, H.; Godbout, N.; Guan, J.; Jamorski, C.; Leboeuf, M.; Malkin, V.; Malkina, O.; Nyberg, M.; Pedocchi, L.; Sim, F.; Triguero, L.; Vela, A. StoBe2004, Version 2.0; 2.0 ed., 2004.

(30) Flükiger, P.; Lüthi, H. P.; Portmann, S.; Weber, J.; Computing, S. C. f. S. MOLEKEL 4.0; 4.0 ed., Manno, Switzerland, 2000.

(31) Borden, W. T. Modern molecular orbital theory for organic chemists; Prentice Hall: Englewood Cliffs, NJ, 1975.

(32) Sapse, A.-M. Molecular orbital calculations for aminoacids and peptides; Birkhäuser: Boston, MA, 2000.

(33) Stöhr, J. NEXAFS spectroscopy; Springer-Verlag: New York, 1992. 